\title{
81. Low Temperature Annealing Stages of Irradiated Copper
}

\author{
By Ryukiti R. HASIGUTI \\ Department of Metallurgy, University of Tokyo \\ (Comm. by T. Mishima, M.J.A., June 13, 1960)
}

1. Introduction. The behaviors of lattice defects introduced into noble metals such as copper, gold, and silver by electron, deuteron, or neutron irradiations have been extensively investigated by many workers. ${ }^{1)}$ Copper is especially well investigated among these metals from both experimental and theoretical standpoints. ${ }^{1)}$ Nevertheless several most important problems are not yet solved. One of these important unsolved problems is the low temperature annealing stage problem of irradiated copper.

Annealing of lattice defects in copper takes place in five successive temperature stages. ${ }^{1-3)}$ The stage I ranges from about $14^{\circ} \mathrm{K}$ to about $65^{\circ} \mathrm{K}$. The stage II has so wide a range that it is often called a garbage annealing stage. It extends from the end of stage $I$ to the advent of stage III. The stage III has a fairly sharp range, which extends from about $240^{\circ} \mathrm{K}$ to about $300^{\circ} \mathrm{K}$. The temperature range of stage IV is considerably different according to different authors, but it extends usually from about $100^{\circ} \mathrm{C}$ to about $200^{\circ} \mathrm{C}$. The stage $\mathrm{V}$ is usually found in the range from about $300^{\circ} \mathrm{C}$ to about $400^{\circ} \mathrm{C}$.

As far as the stage $\mathrm{V}$ is concerned, the interpretations by different authors agree with each other. We shall omit, therefore, to consider the stage V. The interpretations of lower four stages are quite confusing today.

The present author will propose in this paper a new interpretation which explains the lower four stages consistently. Interstitial atoms trapped by impurity atoms will play important roles in the interpretation of stages II and III.

Earlier interpretations of the annealing stages appeared in literatures up to 1955 were reviewed and tabulated by Glen. ${ }^{4)}$ Interpretations appeared since 1956 will be summarized in Table I. The last row of the table shows the interpretation proposed by the present author in this paper.

2. Trapping of an interstitial by an impurity atom. Impurity trapping of interstitials is by no means a new concept. It was first discussed by Lomer and Cottrell ${ }^{11)}$ in 1955. It was not, however, on the firm foundation of knowledges that they considered the impurity trapping of interstitialcies, because important information, which we 
Table I. Interpretations of annealing temperature stages by different authors

\begin{tabular}{|c|c|c|c|c|}
\hline Annealing stages & I & II & III & IV \\
\hline Seeger ${ }^{2)}(1958)$ & $\begin{array}{l}c . p . \\
m . r .\end{array}$ & $\begin{array}{l}c . p . p \\
\left(V_{2}\right)\end{array}$ & $I$ & $V$ \\
\hline Van Bueren ${ }^{3)}(1957)$ & $\begin{array}{l}c . p . \\
m . r .\end{array}$ & $I$ & $V_{2}$ & $V$ \\
\hline A.I. group $^{5)}(1956)$ & c.p. & $\begin{array}{l}c . p . \\
\left(V_{m}\right)\end{array}$ & $I$ & $\begin{array}{l}V, I_{m} \\
I^{*}, V^{*}\end{array}$ \\
\hline G.E. group ${ }^{6)-8)}$ (1959) & $c . p$. & $\begin{array}{l}I^{*} \\
\left(V_{2}\right)\end{array}$ & $V(?)$ & $(?)$ \\
\hline Illinois group 9110$)$ (1956) (1958) & $\stackrel{c . p .}{I}$ & $V_{m}$ & $V$ & - \\
\hline Present author & c.p. & $\begin{array}{c}I^{*} \\
\left(V_{2}, I_{m}\right)\end{array}$ & $I^{* *}$ & $V$ \\
\hline
\end{tabular}

c.p.: close-pair, m.r.: micro-recrystallization, $I$ : interstitial, $V$ : vacancy, $V_{2}$ : divacancy, $V_{m}$ : multi-vacancy, $I_{m}$ : multi-interstitial, $V^{*}$ : trapped vacancy, $I^{*}$ : trapped interstitial, $I^{*}$ in the lowest row: shallow trapped interstitial, $I^{* *}$ : deep trapped interstitial

have today, ${ }^{6)-10)}$ concerning the behaviors of interstitials in stage I annealing processes was lacking at that time. Many authors, who treated the annealing stage problem since 1956, rather disregarded the important roles of impurity trapped interstitials, although they considered, so to say, the auxiliary functions of these trapped interstitials, as some examples are seen in Table I.

Now we shall consider the energy of interaction, $E_{\text {int }}$, between an interstitial atom and an impurity atom. It can be expressed by

$$
\begin{aligned}
E_{\mathrm{int}} & =-\frac{A}{r_{1}^{3}} \cdot \frac{1}{\left(r / r_{1}\right)^{3}+m\left(r / r_{1}\right)}, \\
A & =\frac{15 d}{8 \pi \gamma^{2}} \Delta v_{1} \Delta v_{2} \Gamma, \\
r & =3(1-\sigma) /(1+\sigma), \\
\Gamma & =\frac{2}{5}-2\left(l^{2} m^{2}+m^{2} n^{2}+n^{2} l^{2}\right)
\end{aligned}
$$

where $r$ is the distance between an interstitial atom and an impurity atom, $r_{1}$ and $m$ are appropriate constants, $d$ is a constant with negative value in the case of metals which is derived from elastic constants, $\Delta v$ is the dilatation in a body with a free surface, $\sigma$ is Poisson's ratio, and $l, m$, and $n$ are direction cosines.

The equation (1) reduces to Eshelby's elasticity equation, ${ }^{12)}$ when the distance becomes large compared with the atomic spacing.

An attractive interaction is expected from both negative and positive values of $\Delta v_{1} \Delta v_{2}$, because the value of $\Gamma$ can either be positive or negative according to the value of direction cosines. This means an interstitial is trapped by an oversize impurity atom as well as by an undersize impurity atom. 
Table II. Various configurations of impurity-atom-interstitial pairs in the case of undersize beryllium atoms in copper crystal

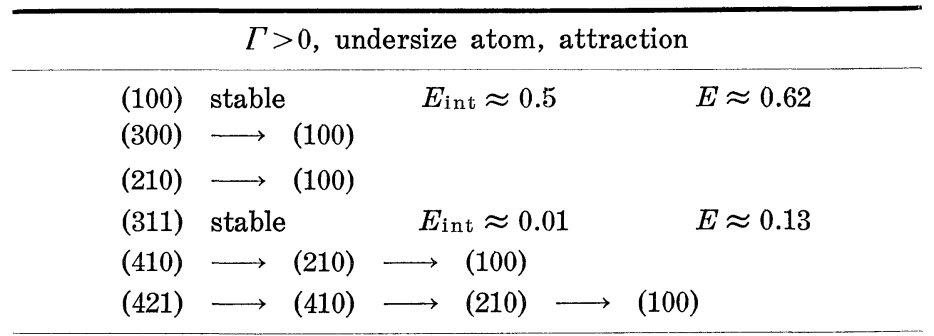

Table III. Various configurations of impurity-atom-interstitial pairs in the case of oversize gold atoms in copper crystal

\begin{tabular}{lll}
\hline \multicolumn{4}{c}{$\Gamma<0$, oversize atom, attraction } \\
\hline$(111)$ stable & $E_{\text {int }} \approx 0.16$ & $E \approx 0.28$ \\
$(221)$ stable & $E_{\text {int }} \approx 0.03$ & $E \approx 0.15$ \\
$(320)$ stable & $E_{\text {int }} \approx 0.003$ & $E \approx 0.12$ \\
$(322) \longrightarrow(221)$ & \\
$(331)$ stable & $E_{\text {int }} \approx 0.01$ & $E \approx 0.13$ \\
$(430) \rightarrow(320)$ & \\
$(333) \longrightarrow(331)$ & & \\
$(432) \longrightarrow(322)$ & $\longrightarrow(221)$ & \\
\hline
\end{tabular}

Now the interaction energies of various impurity-atom-interstitial $\left(A_{i}-I\right)$ configurations are calculated by means of the equation (1). The results are shown in Tables II and III. The indices in the tables indicate the co-ordinates of possible interstitials, a substitutional impurity atom being placed at the origin. The co-ordinates are expressed in unit of $a / 2$, where $a$ is the lattice constant. Thus (311) means, for example, an interstitial is trapped at (311) position, when an impurity atom is at (000).

3. Stable and unstable configurations. Various $A_{i}-I$ configurations are shown in Tables II and III. We have to distinguish between stable and unstable configurations as follows. Let us first examine an undersize impurity atom, which attracts an interstitial, if $\Gamma^{\prime}$ is positive. (100) configuration is of course stable, because it is the closest pair with attractive force.

It is usually considered that an interstitial moves always in $<100>$ direction. ${ }^{10)}$ This means that an interstitial makes interstitialcy movements, when it migrates through the crystal lattice. In other words it has the "C configuration" of Huntington ${ }^{13)}$ as the saddle point, when it makes migration through the lattice. This restriction of the migrating direction is a condition for considering stable configurations.

Now the (300) configuration will be considered. An interstitial at 
(300) position will move towards the impurity atom by the attractive force, resulting in an (100) configuration. Thus the (300) is an unstable configuration. The results of this kind of considerations are shown in Table II for an undersize impurity atom with $\Gamma>0$. It is seen that (300), (210), (410), (421), etc. are all unstable, all reducing to (100) configuration. The only one stable position other than (100) is (311), which has $E_{\mathrm{int}} \approx 0.01 \mathrm{eV}$. (311) is stable, because (111) is a repulsive position.

It should be emphasized here, however, that the occurrence of (311) is rather seldom, because it can be reached only from limited positions.

It may be concluded after all that (100) is almost the unique stable configuration for an undersize impurity atom.

The activation energy of annealing process due to the dissociation of pairs of undersize beryllium atoms and interstitials $\left(A_{i(\mathrm{Be})}-I\right.$ pairs $)$ is $0.62 \mathrm{eV}$ as shown in Table II. This is the stage III annealing process. Dissociated interstitials disappear very quickly at appropriate sinks.

4. Oversize impurity atoms. The situation for an oversize impurity atom is quite different. The results of considerations along the same line as before are given in Table III for an impurity gold atom, which is an oversize atom. An oversize atom attracts an interstitial, when $\Gamma$ is negative. As are shown in the table, there are four stable configurations. The occurrences of these configurations are comparatively evenly distributed. The values of activation energies range from $0.12 \mathrm{eV}$ to $0.28 \mathrm{eV}$ as shown in Table III.

As are expected from the results in Table III, the annealing stages of irradiated gold-doped copper should extend rather continuously from the end of stage I to some higher temperatures as a result of overlapping of several annealing processes with different activation energies. This type of annealing is found in the experimental results of stage II or garbage annealing process.

5. Conclusions. (1) Both undersize impurity atoms and oversize impurity atoms trap interstitials.

(2) Various configurations of impurity-atom-interstitial pairs were considered.

(3) An undersize impurity atom provides with a deep trap, if the dilatation is large. An undersize atom with a small dilatation gives rise of course to a shallow trap.

(4) An oversize impurity atom furnishes several shallow traps with different interaction energies.

(5) Annealing temperature stages of irradiated copper are interpreted as follows.

(5a) Stage I is considered after G.E. group and Illinois group to be due to the annihilations of close vacancy-interstitial $(V-I)$ pairs and 
free migrating interstitials.

(5b) Stage II is interpreted as due to the liberations and annihilations of several kinds of shallow trapped interstitials $\left(I^{*}\right)$ with different interaction energies. The annihilations of divacancies and the dissociam tions and annihilations of multi-interstitials will take place in this stage, if they exist.

(5c) Stage III is considered to be due to the liberations and annihilations of deep trapped interstitials $\left(I^{* *}\right)$ with almost unique interaction energy.

(5d) Stage IV is considered to be due to the annihilations of vacancies.

(5e) These interpretations are summarized in the last row of Table I.

The details of this research will be published elsewhere.

\section{References}

1) See the following review articles. G. H. Kinchin and R. S. Pease: Rep. Progr. Phys., 18, 1 (1955); J. W. Glen: Adv. Phys., 4, 381 (1955); K. Lintner und E. Schmid: Ergebn. exakt. Naturw., 28, 302 (1955); F. Seitz and J. S. Koehler: Solid State Phys., 2, 305 (1956); See also ref. 2).

2) A. K. Seeger: Second U. N. Int. Conf. Peacef. Uses Atomic Energy, P/998 (1958).

3) H. G. van Bueren: Philips Res. Rep., 12, 1, 190 (1957).

4) J. W. Glen: Adv. Phys., 4, 381 (1955).

5) C. J. Meechan and J. A. Brinkman: Phys. Rev., 108, 1193 (1956).

6) J. W. Corbett, R. B. Smith, and R. M. Walker: Phys. Rev., 114, 1452 (1959).

7) J. W. Corbett, R. B. Smith, and R. M. Walker: Phys. Rev., 114, 1460 (1959).

8) J. W. Corbett and R. M. Walker: Phys. Rev., 115, 67 (1959).

9) F. Seitz and J. S. Koehler: Solid State Phys., 2, 305 (1956).

10) G. D. Magnuson, W. Palmer, and J. S. Koehler: Phys. Rev., 109, 1990 (1958).

11) W. M. Lomer and A. H. Cottrell: Phil. Mag., 46, 711 (1955).

12) J. D. Eshelby: Acta Met., 3, 487 (1955).

13) H. B. Huntington: Phys. Rev., 91, 1092 (1953). 